ISSN: 2379-2922

Volume 5, Issue 1, 2 Pages

\title{
Nurse Practitioner and Physician Incivility in Healthcare
}

\author{
Susan Wynn, BSN, MSN, RN \& Dr. Cheryl Holden, DHSc, MSN, RN-BC \\ Bachelor of Science in Nursing, University of Arkansas Fort Smith, Fort Smith, Arkansas, US. \\ Susie.Wynn@uafs.edu
}

According to the American Nurses Association (ANA) (n.d.), incivility can include rude, discourteous, or disrespectful actions. Research indicates that disrespect can negatively impact communication, collaboration, and can decrease the continuity and quality of patient care (Grissinger, 2017). This incivility is not new to many nurse practitioners; it is something that many nurse practitioners experience as bedside nurses even before they become nurse practitioners (Fida, Laschinger, \& Leiter, 2018). One study specified that $55 \%$ of nurses indicated that a physicians' behavior impacted their nursing decisions and that older nurses acknowledged the negative interactions but the younger nurses were affected in a more negative manner (Siedlecki \& Hixson, 2015). Sometimes uncomfortable tensions linger in the nurse's station and the disrespectful glances in the hallway are almost unbearable for many nurse practitioners.

The lack of respect many nurse practitioners feel when in the presence of some physicians is very real. Many nurse practitioners are categorized as less educated or not having the knowledge physicians do in order to prescribe, diagnose, or care for patients in health care settings. According to Gary Edwards, M.D., many nurse practitioners lack the clinical experiences or professional lens to function as nurse practitioners (G. Edwards, personal communication, August 26, 2019). This attitude often creates tensions and disrespect that can be interpreted as incivility. Sometimes the backbiting, belittling, and whispering of words, negative looks, and gossip are issues nurse practitioners find challenging. While it is true that some nurses who pursue becoming a nurse practitioner have few years of clinical nursing experience, it must also be noted that many nurse practitioners have years of clinical experience caring for patients at the bedside and have introduced positive changes in healthcare environments (Ryder, Jacob, \& Hendricks, 2019).

This unprofessionalism and incivility associated with these fore-mentioned issues is an unfortunate picture of reality that must be addressed and cannot be avoided. Many nurses have provided total care to hospital patients for twelve hours daily prior to becoming nurse practitioners. One study indicated that intensive care physicians spend little amounts of time with patients (Butler, et al., 2018). From experience, this finding is the norm in many clinical environments. For example, the physician shows up on his or her rounds for a short amount of time and continuously picks the nurse's brain to find out what the patient's current status.

Many nurse practitioners have had years of bedside experience dealing with both acute and chronic patients. Nurse Practitioners deserve the respect and belief that they are knowledgeable practitioners. For example, many nurses with clinical knowledge and experience use critical thinking to divert code situations before the physician arrives and intervenes on the patient's behalf. These nurses who intervene and many times save the patient's life become clinical nurse practitioners. We must remember nurses are amongst one of the most honest and trusted professions nationwide; therefore, it is sensible to understand that in many instances nurse practitioners have better bedside rapport and mannerisms with patients and gain a patient's trust much easier than some physicians who can be less than personable at times (Norful, de Jacq, Carlino, \& Poghosyan, 2018). However, this does not mean that nurse practitioner should be favored over a physician. It simply means they deserve respect because of their contribution to the medical profession and to patients. A nurse practitioner can work in harmony with a physician by co-managing patient care and realizing that each of us has valuable 
contributions to the medical profession without implementing or participating in incivility (Norful, de Jacq, Carlino, \& Poghosyan, 2018).

The fact is, healthcare professionals provide care to patients in diverse healthcare environments. If there is not peace and comradery amongst nurse practitioners and physicians, the medical profession as a whole may suffer because some nurse practitioners may leave the profession (Fida, Laschinger, \& Leiter, 2018). However, more importantly, the patients may suffer from the discourse, tensions, and incivility that occurs in healthcare environments. Therefore, going back to the basics, common courtesy includes having respect for one another for the sake of preserving our profession and our patients. Respect for each other as professionals is not difficult. Also, respect for each other's education is not difficult, respect that working together as a team can produce better outcomes for patients and for the overall healthcare environment is not difficult to comprehend. If respect, compassion for one another, kindness, and a team mindset is adopted, there is a chance that the incivilities that exist between nurse practitioners and physicians may begin to decrease over time leading to improved work and an patient care environments.

\section{REFERENCES}

American Nurses Association (n.d.). Violence, incivility, and bullying. Retrieved from https://www.nursingworld. org/practice-policy/work-environment/violence-incivility-bullying/.

Butler, R., Monsalve, M., Thomas, G.W., Herman, T., Segre, A.M., Polgreen, P.M., \& Suneja, M. (2018). Estimating time physicians and other healthcare workers spend with patients in an intensive care unit using a sensor network. The American Journal of Medicine, 131(8), 972. doi:10.1016/j.amjmed.2018.03.015.

Gibson, A. (2017). [Update] Dr.'s post on nurse practitioners goes vital, claims someone else posted. Retrieved fromhttps://nurse.org/articles/doctor-calls-nurse-practitioners-minions/

Grissinger, M. (2017). Disrespectful behavior in health care. Its impact, why it arises and persists, and how to address it- Part 2. Pharmacy \& Therapeutics, 42(2), 74-75, 77. Retrieved from https://www.ncbi.nlm.nih. gov/pmc/articles/PMC5265230/.

Fida, R., Laschinger, H.K.S., \& Leiter, M.P. (2018). The protective role of self-efficacy against workplace incivility and burn out in nursing. Health Care Management Review, 43(1), 21-29. doi: 10.1097/ HMR0000000000000126.

Norful, A.A., de Jacq, K., Carlino, R., Poghosyan, L. (2018). Nurse practitioner-physician co management: A theoretical model to alleviate primary care strain. Annals of Family Medicine, 16(3), 250-256. doi: 10.1370/ afm.2230.

Ryder, M., Jacob, E., \& Hendricks, J. (2019). An inductive qualitative approach to explore nurse practitioners views on leadership and research: An international perspective. JCN, 28(13-14),2644-2658.doi:10.1111/ jocn.14853.

Siedlecki, S., Hixon, E. (2015). Relationships between nurses and physicians matter. OJIN, 20(3). doi: 10.3912/ OJIN.Vol20No03PPT03.

Citation: Susan Wynn, Dr. Cheryl Holden, "Nurse Practitioner and Physician Incivility in Healthcare". American Research Journal of Nursing. 2019; 5(1): 1-2.

Copyright @ 2019 Susan Wynn, Dr. Cheryl Holden, This is an open access article distributed under the Creative Commons Attribution License, which permits unrestricted use, distribution, and reproduction in any medium, provided the original work is properly cited.

American Research Journal of Nursing

Page 2 